\title{
Viewpoint
}

\section{Revisiting Zika (and Rubella)}

\author{
Adolfo Martinez-Palomo \\ Center for Advanced Studies, Molecular Pathogenesis, Avenida IPN 2508, \\ o 030 Mexico City, DF, Mexico \\ E-mail: amartine@cinvestav.mx
}

\begin{abstract}
Three months after the World Health Organization declared the epidemic of Zika virus infections to be a Public Health Emergency of International Concern, we can look back at what we have learned and prospects for controlling the disease. Although Zika virus infections may explain many cases of brain damage in newborns, it may not be the only cause. We need a clear association between confirmed cases of Zika infections in pregnant women and microcephaly in newborns. Until we reach a firm conclusion, past experience with another virus that causes damage to newborns offers some hope. The development and almost universal use of rubella vaccine has all but eliminated the congenital rubella syndrome in the world. Rapid development of Zika virus vaccine might well do the same for this epidemic.
\end{abstract}

Journal of Public Health Policy online publication, I 5 June 20I6; doi:I0.I057/S4I 27I-0I 6-000I-8

Keywords: Zika virus; dengue; chikungunya; rubella vaccine; microcephaly

It has been three months since WHO declared the Zika epidemic a

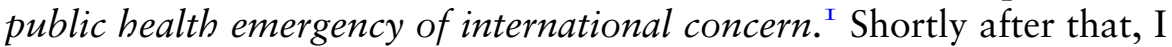
commented on the epidemic in this journal. ${ }^{2}$ Infections have occurred in 35 countries in the Americas, but the problem is concentrated in Brazil where health authorities have reported nearly ı००,০০০ suspected cases of Zika. How big is Brazil's Zika outbreak? We do not know, because health authorities report only suspected, not confirmed cases. I learned about what was reported by consulting the websites of the U.S. Centers for Disease Control and Prevention, the Boletines Epidemiológicos de los Ministerios de Salud de Brazil, and comparable websites in Colombia and México on 9th May, 2016.

The Zika epidemic is frightening because of clinical findings seen in babies born to mothers who had the infection during pregnancy. Newborns manifest brain malformations, mainly microcephaly, that appear to be linked to Zika infections. To understand the epidemic, it is important to assess the real number of babies with cerebral damage. 
For the first four months of 2016 , Brazil initially reported 7,343 probable cases of microcephaly. Only I27I were later confirmed. In other countries for the same period, the picture was different. In Colombia only five cases of microcephaly have been reported. There were 3,75I confirmed Zika infections. In Mexico, from a total of 264 confirmed Zika infections, 74 in women, no microcephaly cases have occurred. In the U.S. there are no reports of brain malformations linked to 472 Zika infections acquired abroad, nor in 629 infections reported in Puerto Rico.

For Brazil, there is no doubt that Zika is a serious public health problem. The difficulty in identifying Zika virus infections in patients makes the challenge greater. Clinically, it is virtually impossible to differentiate a Zika infection from dengue or chikunkunya, two other viral infections transmitted by the same Aedes mosquito. And in Brazil, these diseases are more common than Zika. Since the start of 2016, more than 800,000 possible cases of dengue and 40,000 of chikunkunya have occurred in Brazil.

Making matters more difficult, women likely to be infected with the Zika virus live in low resource regions, where they cannot easily comply with the recommendation to avoid biting mosquitoes. They do not have access to reproductive health measures, in particular legal and medically safe abortions.

So the key question remains unanswered: what percentage of pregnant women infected with Zika during pregnancy will deliver babies with irreversible brain malformations? A recent study in Rio de Janeiro of 42 pregnant mothers infected with the Zika virus uncovered serious complications in I 2 of them. Almost one-third of the women in that small group had serious problems - from fetal death, to insufficient fetal growth, brain malformations, and placenta damage. ${ }^{3}$

Less frequently (probably in one out of 4000-8000 infections), the Zika virus produces reversible paralysis (Guillain-Barré syndrome) in the infected individual who may require hospitalization.

What are the other possible causes of the recent appearance of microcephalies? Could insecticides used to control Aedes mosquitos, in particular pyriproxyfen, a pyridine-based pesticide, alter pregnancy or fetal development? Both WHO and the U.S. Environmental Protection Agency have ruled out this possibility. Yet other worries persist. Could release of genetically modified mosquitoes or those sterilized by low doses of radiation be causing the problem? Experts have discarded this 
hypothesis. Finally, the WHO Global Advisory Committee on Vaccine Safety concluded that the use of various vaccines in the affected regions is not related to the appearance of microcephaly in babies.

Can we learn from the world's experience with another virus, one that causes birth defects when mothers are infected early in pregnancy? Infection with rubella during the first ten weeks of pregnancy may produce adverse effects in fetal development in up to 90 percent of pregnancies. The risk is much lower after the 28th week of pregnancy. Rubella causes many congenital defects, including cataracts, congenital heart disease, hearing impairment, and developmental delay - together the congenital rubella syndrome.

During the I962-I965 global rubella pandemic, an estimated I2.5 million rubella cases occurred in the United States, resulting in 2000 cases of encephalitis, II,250 therapeutic or spontaneous abortions, 2100 neonatal deaths, and 20,000 infants born with congenital rubella syndrome. Use of the rubella vaccine dramatically lowered these numbers; at present fewer than ten cases of rubella are reported each year in the U.S.

The two billion dollars requested by president Obama will surely stimulate development of a Zika vaccine in the near future and it may well be the solution to the epidemic, just as the vaccine ended epidemic rubella. Until we have a vaccine, health authorities in all affected countries are waiting to learn when, how, and with what frequency the Zika virus produces irreversible damage in newborns when pregnant women are infected. With such information, health measures can be based on scientific knowledge not on panic. WHO probably exaggerated the character of the Zika epidemic by labeling it an "international concern." The media, presenting frightening pictures of microcephalic babies, has continued to refer to the infection as "scary."

Submitted on 9th May 2016.

\section{About the Author}

Adolfo Martinez Palomo, MD, Sc.D., is a cell biologist working on infectious diseases research at the Center for Advanced Studies, Mexico City. He has carried out research on the biology of parasitic diseases common in Mexico, such as amebiasis, giardiasis, onchocerciasis, and Chagas disease, and participated in many national and international policy making bodies. 


\section{References}

I. WHO statement I February 2016.

2. Martinez-Palomo, A. (20I6). Zika virus: An international emergency? Journal of Public Health Policy (2016) 37: I33-I35. doi:I0.I057/jphp.20I6.II; published online 25 February 2016.

3. Brasil, P., Pereira, J. P., et al (20I6) Zika virus infection in pregnant women in Rio de JaneiroPreliminary Report, New England Journal of Medicine March 4, 20I6, at NEJM.org. doi:Io. I056/NEJMoaI6024I 2. 